\title{
Renoprotective Action of a Matrix Metalloproteinase Inhibitor in Progressive Mesangioproliferative Nephritis
}

\author{
Takayuki Kuroda Masao Masui Mitsuru Notoya Masashi Ito \\ Yoshinori Tamura Hiroyuki Okamoto Eri Kanaoka \\ Toshihiro Shinosaki
}

Shionogi and Co., Ltd., Toyonaka City, Japan

\section{Key Words}

Matrix metalloproteinase-2 - Glomerulonephritis · Glomerular basement membrane •

Glomerular epithelial cells · Extracellular matrix

\begin{abstract}
Background/Aim: Matrix metalloproteinases (MMPs) play pivotal roles in extracellular matrix turnover and are involved in chronic kidney disease. The renoprotective action of a synthetic MMP inhibitor, compound A, was investigated in chronic nephritis. Methods: Nephritis was induced by a single injection of anti-Thy1.1 antibody to unilaterally nephrectomized rats. The effects of compound A on proteinuria, blood urea nitrogen, and matrix-related gene expressions were evaluated. Collagen accumulation, as assessed by periodic acid-Schiff staining and hydroxyproline content, was determined. The integrity of glomerular epithelial cells and glomerular basement membrane was evaluated with desmin immunohistochemistry and electron microscopic detection of anionic charge sites, respectively. Results: Treatment with compound A notably attenuated proteinuria, ameliorated blood urea nitrogen, and prevented glomerulosclerosis. Gene upregulation of collagen and transforming growth factor $\beta 1$ in the cortex was prevented in the treated animals. Glomerular epithelial cell injury was milder, and glomerular basement membrane anionic sites were protected with the treatment. Conclusion: A novel MMP inhibitor, compound $A$, exerts protective effects in progressive glomerulonephritis. Compound $A$ ameliorates various aspects of renal injuries and may have therapeutic potential toward kidney diseases.

Copyright $\odot 2012$ S. Karger AG, Basel
\end{abstract}




\section{Introduction}

Matrix metalloproteinases (MMPs) are a large family of zinc-dependent proteolytic enzymes which degrade various kinds of extracellular matrix (ECM) components. Twentyeight members of the MMP family have been identified [1]. They are classified into four subgroups including gelatinases, interstitial collagenases, stromelysins, and the membrane-type MMPs (MT-MMPs). MMPs and their inhibitors, i.e. tissue inhibitors of metalloproteinase, are presumed to be implicated in the progression of chronic kidney disease (CKD) by affecting ECM metabolism [2-5]. However, the precise pathological roles of these proteins in chronic renal disorders are not fully elucidated [1]. Since the accumulation of ECM represents an important pathological feature in the development of glomerulosclerosis and tubulointerstitial fibrosis, there is little doubt that an impairment of the balance of ECM turnover, such as a decrease in matrix degradation or an enhancement of matrix synthesis, contributes to the progression of CKD. In general, MMP activity may counteract accumulation of ECM, thereby prevent CKD progression. However, on the contrary, mounting evidence has suggested that some MMPs may contribute to a worsening of kidney diseases [6, 7].

MMP-2 is a gelatinase enzyme and has activity of degrading type IV collagen, a major component of the basement membrane. Pro-MMP-2 is activated by MT1-MMP and plasmin $[8,9]$. MMP-2 is suggested to play a role in tubular cell epithelial-mesenchymal transition (EMT), a process by which differentiated epithelial cells undergo a phenotypic conversion to matrix-producing fibroblasts and myofibroblasts, degrade the tubular basement membrane, and generate interstitial fibrosis [10]. Transgenic expression of MMP-2 in tubular epithelial cells (TECs) leads to structural changes of the tubular basement membrane and phenotypic changes of TECs, two of the processes implicated in EMT [11]. In a canine disease model, MMP-2 expression is upregulated and localized in the area of the splitting tubular membrane, suggesting MMP-2 involvement in EMT [12].

MMP-2 may also be involved in glomerular injury. After injury, increased activity of MMP-2, which is secreted by mesangial cells (MCs) in glomeruli and leukocytes penetrating into the glomerular basement membrane (GBM), results in the degradation of the GBM [1315]. We have previously shown that upregulation of MMP-2 in glomeruli contributes to glomerular damage by altering the GBM component in nephritic rats [16]. Furthermore, in patients with CKD, increased MMP-2 expression in glomeruli and a significant correlation between circulating MMP-2 activity and serum creatinine concentration have been reported $[17,18]$.

Transforming growth factor (TGF)- $\beta 1$ is a multifunctional cytokine which regulates ECM metabolism and has been shown to play a pivotal role in the development of irreversible kidney disease $[19,20]$. The possible involvement of TGF- $\beta$ in the progression process of CKD includes a decrease in MMP synthesis and an enhancement of tissue inhibitors of metalloproteinase production $[1,21]$. Unlike expression of other MMPs, however, MMP-2 expression is stimulated by TGF- $\beta$ in vitro [22]. The enzymatic activity of MMP-2 is increased during kidney disease, suggesting its detrimental aspect to kidney disease $[3,23]$. In rats with acute glomerulonephritis, treatment with BB-1101, a non-selective MMP inhibitor, ameliorates massive proteinuria [24]. However, it remains uncertain whether or not the blockade of the enzymatic activity of MMP-2 is beneficial in the treatment of chronic glomerulonephritis.

Compound $\mathrm{A}$ is a newly synthesized carbonyl acid derivative with potent and selective inhibition against MMPs, especially MMP-2 and MMP-8. This compound exerts little inhibition on MMP-1, MMP-3, or MT1-MMP, and is available for oral use. The present study focused on the evaluation of the renoprotective action of the MMP inhibitor compound A in chronic anti-Thyl.1 glomerulonephritic rats. Our findings demonstrate possible therapeutic intervention using this MMP inhibitor for the treatment of progressive kidney injury. 
Fig. 1. Structure and concentration-dependent inhibition of compound A, and plasma concentration-time profile in rats. a Chemical structure of compound A. b Inhibitory dose-response curves of compound A for MMP-1, -2, -3, -8, -9, and -13 and MT1-MMP. c The mean plasma concentrations of compound $\mathrm{A}$ at $0,0.5,1,2,4,6$, and $24 \mathrm{~h}$ after oral administration were evaluated.
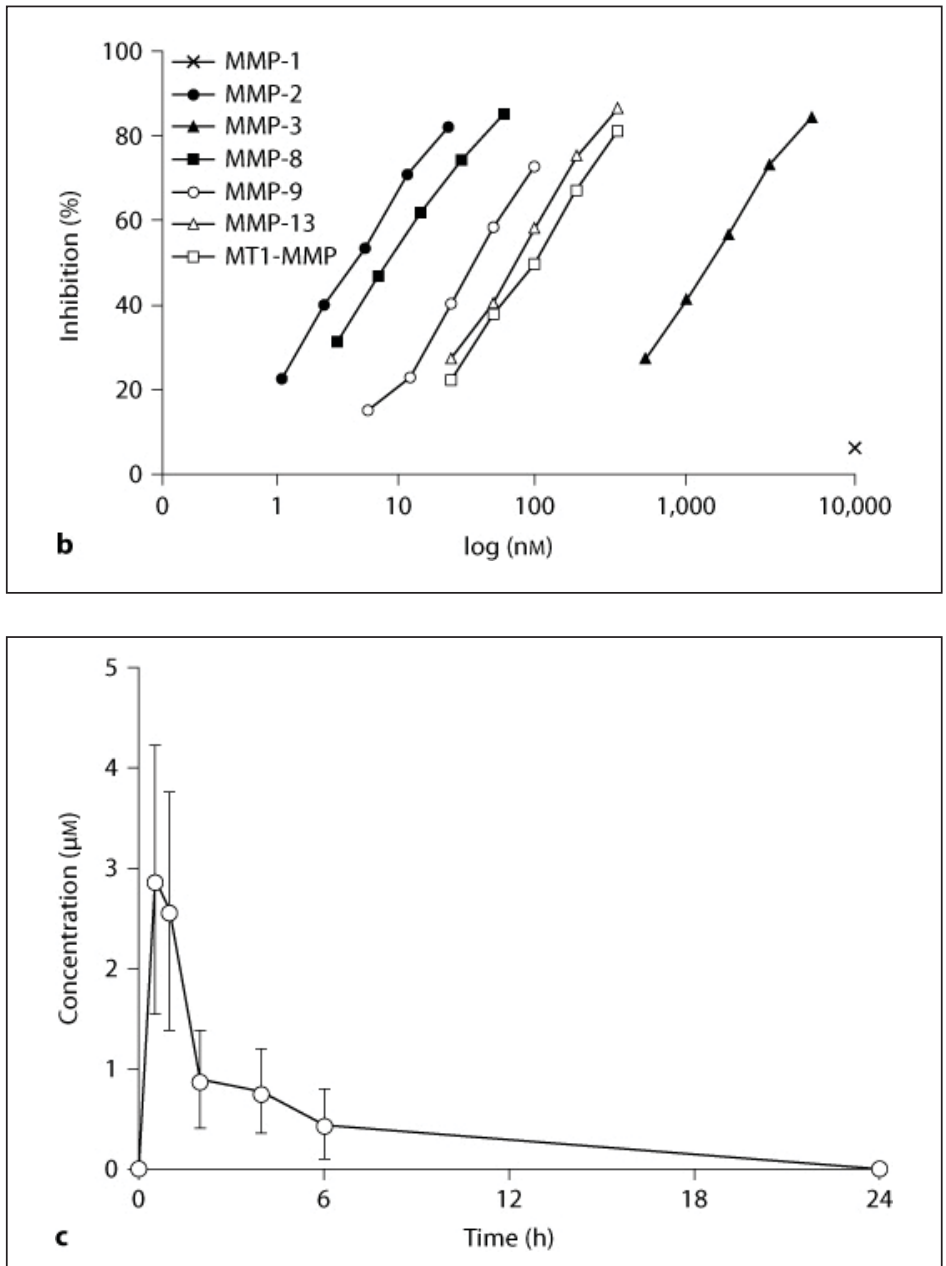

\section{Methods}

\section{Animals and Antibody}

Male Wistar/ST rats were obtained from Japan SLC, Inc. (Shizuoka, Japan). The animals were housed at $25^{\circ} \mathrm{C}, 40-60 \%$ humidity, and in a 12-hour light-dark cycle. They were given free access to standard chow and tap water. All animal experiments complied with the Principles of the Animal Care and Use Committee of Shionogi. Anti-Thyl.1 monoclonal antibody E30 was obtained from ascites fluid of mice [25].

\section{MMP Inhibitor}

$\mathrm{IC}_{50}$ values of MMP inhibition of compound A [(2R)-2-\{5-[4-(ethyl-methylamino)phenyl]-thiophene-2-sulfonylamino\}-3-methyl-butyric acid] (fig. 1a) were determined using purified human MMPs and quenched fluorescent peptide substrates [26]. A pharmacoki- 
netic study of compound A was performed. The drug suspended with $0.5 \%$ methylcellulose solution was orally administered to rats $(\mathrm{n}=6)$ at a dosage of $3 \mathrm{mg} / \mathrm{kg}$, and blood samples were taken at $0,0.5,1,2,4,6$, and $24 \mathrm{~h}$. The blood concentrations of compound A were determined by reverse-phase high-performance liquid chromatography. Pharmacokinetic parameters were calculated using WinNonLin software (Pharsight Inc.).

\section{Pharmacological Study}

Six-week-old Wistar/ST rats were used in this study. Under anesthesia with sodium pentobarbital, the left kidney was removed through a flank incision. Two weeks after nephrectomy, nephritis was induced by a single injection of E30 $(70 \mu \mathrm{g} / \mathrm{rat})$ into the tail vein. The same volume of saline was administered to control rats. The rats were allocated into three groups: control group $(n=3)$, nephritis group $(n=7)$, and compound A treatment group $(\mathrm{n}=7)$. Compound A was orally given at a dose of $3 \mathrm{mg} / \mathrm{kg} 1 \mathrm{~h}$ prior to E30 injection and consecutively administered once a day throughout the experiment. Each animal was kept in a metabolic cage to collect the 24 -hour urine sample at the indicated time points. Plasma samples were collected from the tail vein.

The rats were sacrificed at week 23 . The kidney, liver, spleen, and adrenal were harvested and tissue weights were measured. For histological examination, part of the kidney was fixed in formalin and embedded in paraffin. Sections up to $4 \mu \mathrm{m}$ in thickness were stained with periodic acid-Schiff (PAS) and hematoxylin. For hydroxyproline measurement, renal cortical tissue was frozen with dry ice and kept at $-80^{\circ} \mathrm{C}$.

Among the animals in each group, several rats (control group: $\mathrm{n}=3$, nephritis group: $\mathrm{n}=4$, compound A treatment group: $\mathrm{n}=4$ ) were used for gene expression analysis, electron microscope examination, and immunohistochemistry. The selection of rats was based on the average urinary protein excretion (UPE) at 23 weeks. For gene expression analysis, RNA was isolated from renal cortices and glomeruli. For electron microscope examination, polyethyleneimine was injected via the tail vein at $15 \mathrm{~min}$ before sacrifice to detect anionic charge sites (ACSs) on the GBM. For immunohistochemistry, part of the kidney was fixed in formalin and embedded in paraffin to identify the expression of desmin.

\section{Miscellaneous Measurements}

Urinary protein was determined by the pyrogallol red method, and blood urea nitrogen (BUN) was determined by the urease indophenol method (Wako Chemical Industries Ltd., Osaka, Japan). Plasma total cholesterol, triglyceride, non-esterified fatty acids (NEFA), aspartate transaminase (AST), and alanine transaminase (ALT) were measured by routine methods on a Hitachi-7070 clinical autoanalyzer.

\section{Morphological Study}

Glomerulosclerosis was evaluated with quantitative measurement of the PAS-stained area in glomeruli. For each section, 30 (control group) or 50 (nephritis and treatment groups) randomly selected glomeruli were subjected to computer image analysis (Raizu Co. Ltd., Sendai, Japan). The percentage of glomerular area with PAS staining was calculated as the index of glomerulosclerosis.

Tubulointerstitial injury was defined as inflammatory cell infiltration, tubular dilation, and interstitial fibrosis. Injury was graded semiquantitatively on a scale of $0-4$ as previously described [27]. The average score of 10 fields was determined as the index of tubulointerstitial lesions. 


\section{Determination of Hydroxyproline Content}

The collagen content was assessed by determining total hydroxyproline according to the method of Kivirikko et al. [28]. The results were expressed as hydroxyproline per protein. Protein was determined according to the Lowry method.

Quantitative Analysis of Gene Expression

Real-time polymerase chain reaction (PCR) was performed using the ABI PRISM 7000 Sequence Detection System (Applied Biosystems, Foster City, Calif., USA). The primer pairs and probes were used for real-time PCR detection as previously reported [16].

\section{Detection of ACSs on the GBM}

The detection of ACSs on the GBM was performed as previously reported [29]. The kidney was cut into small pieces and immersed in a mixture of $0.1 \%$ glutaraldehyde and $2 \%$ phosphotungstic acid. The samples were postfixed in $2 \% \mathrm{OsO}_{4}$ buffered with sodium cacodylate buffer. The tissue pieces were rinsed, dehydrated, and embedded in epoxy resin. Ultrathin sections were contrasted with lead citrate and examined with an electron microscope (100-CX; Jeol, Tokyo, Japan).

\section{Immunohistochemistry}

Immunohistochemistry for desmin was carried out as previously reported [27]. The sections were incubated with HRP-conjugated anti-human desmin (DAKO, Glostrup, Denmark) and incubated with diaminobenzidine solution. For each section, 10 glomeruli were assessed based on the degree of staining for desmin. Glomerular desmin was scored semiquantitatively in a blind fashion, according to a scale of $0-3$.

\section{Statistical Analysis}

Values are expressed as the mean and standard error. Student's t test was used. For all experiments, statistical significance was set at $\mathrm{p}<0.05$.

\section{Results}

$I C_{50}$ Values and Pharmacokinetics of Compound $A$

The $\mathrm{IC}_{50}$ values (in nM) of compound A for MMP inhibition were as follows: MMP-1 >10,000, MMP-2 4.2, MMP-3 1,790, MMP-8 15.1, MMP-9 37.4, MMP-13 69.7, and MT1MMP (MMP-14) 1,580 (fig. 1b). Plasma concentrations after orally administered $3 \mathrm{mg} / \mathrm{kg}$ of compound A reached the maximal level of $2.9 \mu \mathrm{M}$ at $0.5 \mathrm{~h}$ and gradually decreased to $0.4 \mu \mathrm{M}$ at $6 \mathrm{~h}$ and became undetectable at $24 \mathrm{~h}$ (fig. 1c). The mean area under the plasma concentration-versus-time curve (AUC $24 \mathrm{~h}$ ) was $11 \mu \mathrm{M} / \mathrm{h}$. The blood concentration of compound A indicated that an oral dosage of $3 \mathrm{mg} / \mathrm{kg}$ compound A was expected to inhibit MMP-2 activity.

Effects of Compound A on UPE, BUN, and Morphological Changes

Effects of compound A on UPE and BUN in nephritic animals are shown in figure 2. UPE exhibited a biphasic increase (fig. 2a). The first increase was rapidly observed within 1 week after antibody injection. After the peak, it showed a transient recovery from the second to the fourth week. UPE increased again from week 4 to the end of the study ( 23 weeks). BUN continuously increased from week 7 after the nephritis induction, accompanied by the increase in UPE (fig. 2b). In control unilaterally nephrectomized rats, the amount of UPE and the level of BUN showed little change. Suppression of UPE in the compound A-treated rats 
Fig. 2. Effect of compound A on UPE and BUN in nephritic rats. a Plots show the mean values and bars the standard errors. Open circles: unilaterally nephrectomized control rats $(n=3)$; closed circles: nephritic rats $(n=7)$; gray circles: rats treated with compound A ( $\mathrm{n}=7)$. b Columns show the mean values and bars the standard errors. White columns: unilaterally nephrectomized control rats $(\mathrm{n}=3)$; black columns: nephritic rats $(\mathrm{n}=7)$; gray columns: rats treated with compound A $(n=7)$. * $p<0.05$ : significantly different from the control group. ${ }^{*} \mathrm{p}<0.05$ : significantly different from the nephritis group.

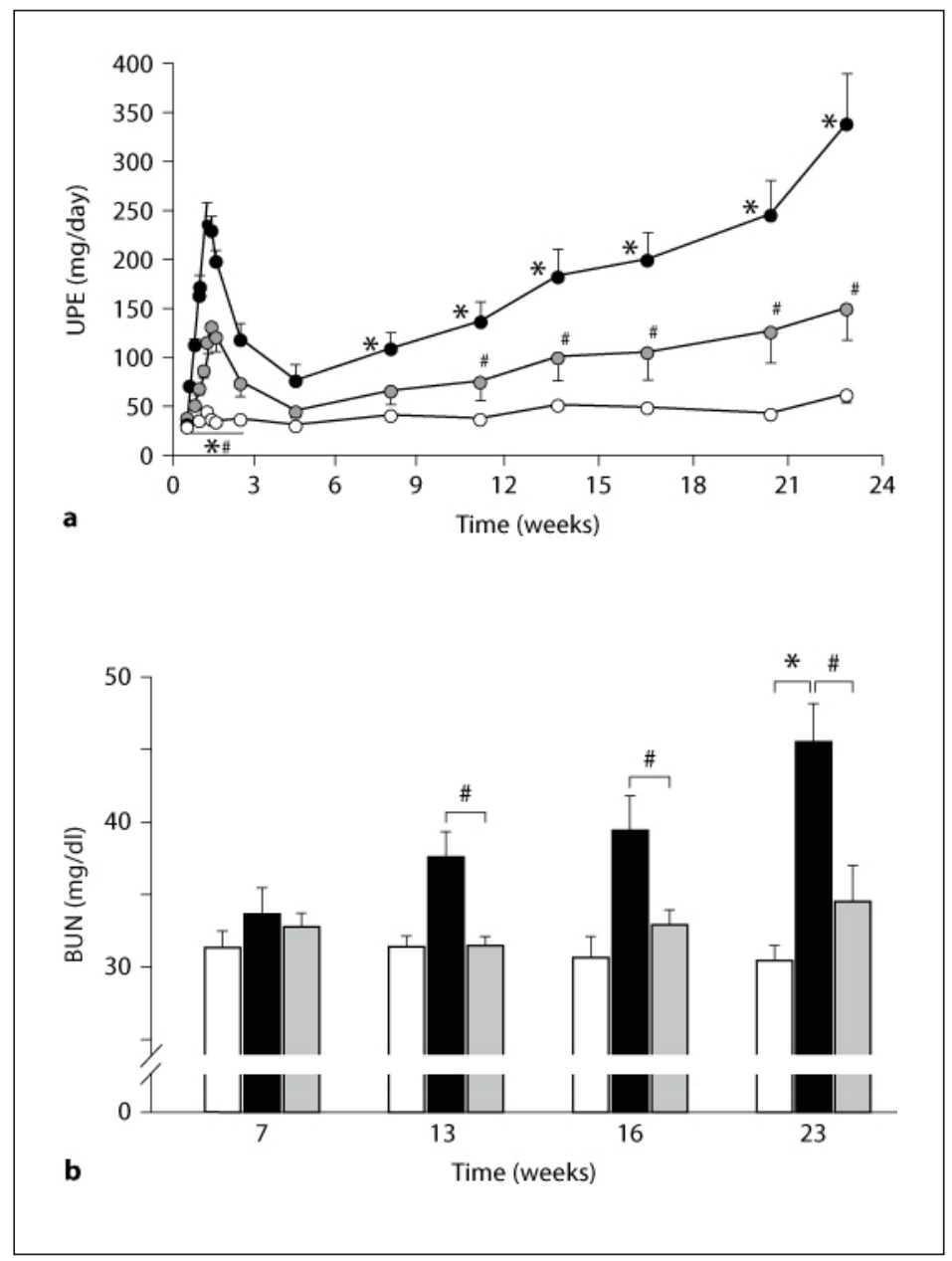

was demonstrated from day 2 to week 23 (fig. 2a). Treatment with compound A significantly decreased BUN values at weeks 13, 16, and 23 (fig. 2b).

Representative morphological changes in kidney tissues of nephritic rats at the end of the study are shown in figure $3 \mathrm{a}-\mathrm{c}$. A marked accumulation of ECM in glomeruli of the nephritis group was observed, showing typical glomerulosclerosis (fig. 3b). Tuft adhesion to Bowman's capsule was frequently observed, suggesting glomerular epithelial cell (GEC) injury. Tubular atrophy and infiltration of inflammatory cells into the interstitial area were detected in the nephritic group. On the other hand, treatment with compound A obviously attenuated these histological changes in the nephritic rats (fig. 3c). Measurement of PAS staining in the glomeruli was examined to evaluate glomerulosclerosis (fig. 3d). The PAS staining index in nephritic rats was significantly higher than that in control rats. Treatment with compound A significantly suppressed glomerulosclerosis. Semiquantitative analysis of kidney damage revealed that the scores of tubulointerstitial injuries were remarkably high in nephritic rats, and treatment with compound A reduced the scores, although not significantly (fig. 3e). The amount of hydroxyproline in the renal cortex was determined to assess the effect of compound A on collagen contents in the kidney tissue (fig. 3f). Hydroxyproline contents in nephritic rats significantly increased compared with those in control rats. Treatment with compound A significantly attenuated the increase in hydroxyproline in the nephritic rats. These results corresponded to the findings from the morphological studies. 

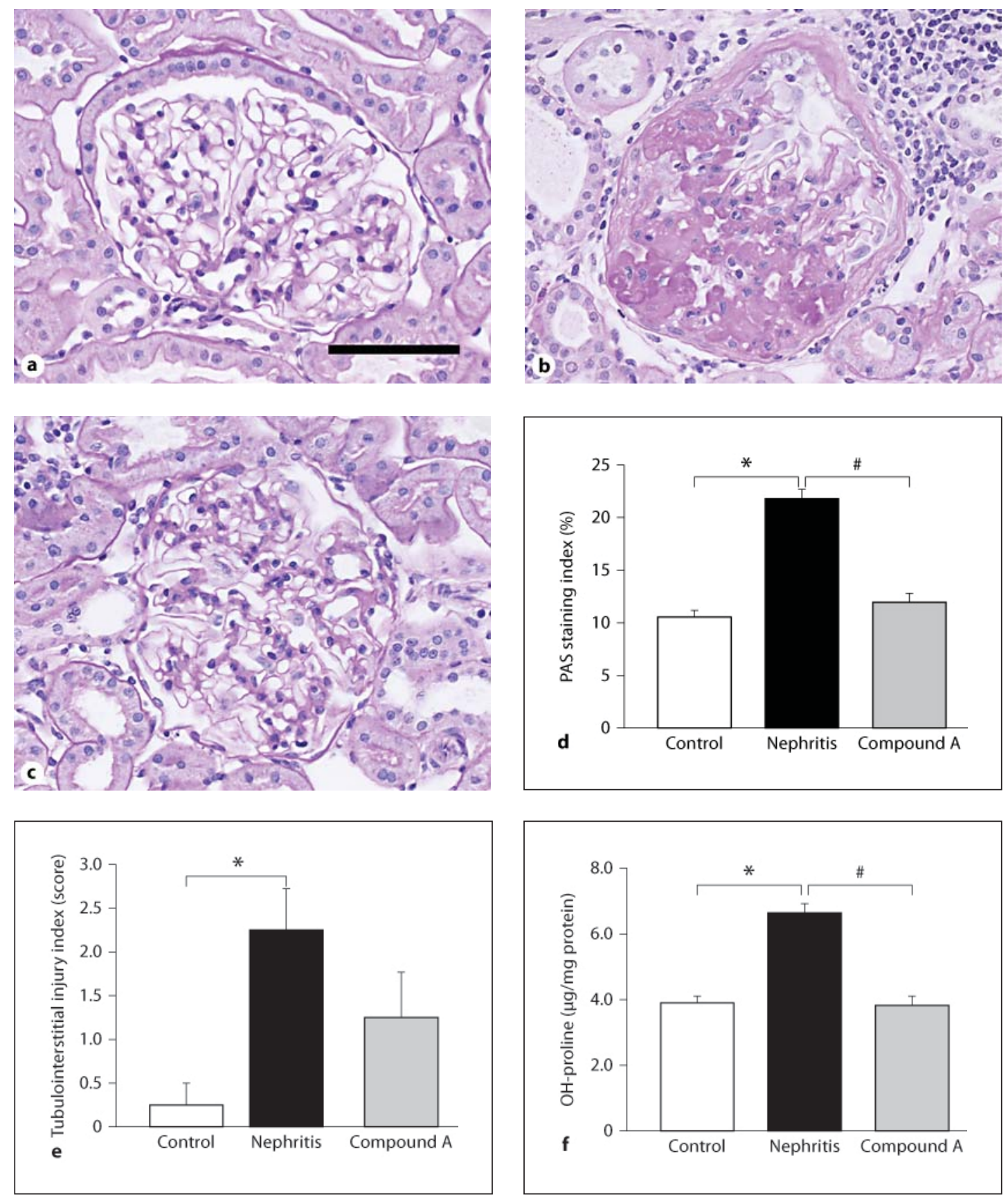

Fig. 3. Effect of compound A on glomerulosclerosis and tubulointerstitial injury in nephritic rats. a-c Light microscopy of kidney tissues stained with PAS. a Unilaterally nephrectomized control rat. b Nephritic rat. A single injection of E30 to unilaterally nephrectomized rats induced deposition of ECM in glomeruli and tubulointerstitial fibrosis. c Treatment with compound A. Drug treatment suppressed the morphological changes in both glomeruli and the tubulointerstitial area. Scale bar $=50 \mu \mathrm{m}$. d PAS-stained area in glomeruli was measured using computer image analysis to evaluate glomerulosclerosis. e Semiquantitative analysis of tubulointerstitial injury. $\mathbf{f}$ Hydroxyproline contents in renal cortices. d-f Columns show the mean values and bars the standard errors. White columns: unilaterally nephrectomized control rats $(\mathrm{n}=3)$; black columns: nephritic rats $(\mathrm{n}=7)$; gray columns: rats treated with compound $\mathrm{A}(\mathrm{n}=7) .{ }^{*} \mathrm{p}<0.05$ : significantly different from the control group. ${ }^{*} \mathrm{p}<0.05$ : significantly different from the nephritis group. 
Table 1. Effect of compound A on tissue weight in chronic Thy-1.1 nephritic rats

\begin{tabular}{lllll}
\hline & $\begin{array}{l}\text { Kidney } \\
\text { g/kg BW }\end{array}$ & $\begin{array}{l}\text { Liver } \\
\text { g/kg BW }\end{array}$ & $\begin{array}{l}\text { Spleen } \\
\text { g/kg BW }\end{array}$ & $\begin{array}{l}\text { Adrenal } \\
\text { g/kg BW }\end{array}$ \\
\hline Control $(\mathrm{n}=3)$ & $5.36 \pm 0.46$ & $25.7 \pm 0.5$ & $1.29 \pm 0.01$ & $0.098 \pm 0.003$ \\
Nephritis $(\mathrm{n}=7)$ & $7.90 \pm 0.64^{*}$ & $29.7 \pm 0.8^{*}$ & $1.61 \pm 0.05^{*}$ & $0.115 \pm 0.003^{*}$ \\
Compound A $(\mathrm{n}=7)$ & $6.47 \pm 0.49$ & $27.0 \pm 0.8^{*}$ & $1.35 \pm 0.06^{*}$ & $0.109 \pm 0.005$ \\
\hline
\end{tabular}

Tissue samples from unilaterally nephrectomized rats (Control), nephritic rats (Nephritis), and rats treated with compound A (Compound A) were collected. Data represent the mean and standard error. $\mathrm{BW}=$ Body weight. ${ }^{*} \mathrm{p}<0.05$ : significantly different from the control group. ${ }^{*} \mathrm{p}<0.05$ : significantly different from the nephritis group.

Table 2. Plasma lipid, AST, and ALT levels at the end of the study

\begin{tabular}{|c|c|c|c|c|c|}
\hline & $\begin{array}{l}\mathrm{TC} \\
\mathrm{mg} / \mathrm{dl}\end{array}$ & $\begin{array}{l}\mathrm{TG} \\
\mathrm{mg} / \mathrm{dl}\end{array}$ & $\begin{array}{l}\text { NEFA } \\
\mu E q / 1\end{array}$ & $\begin{array}{l}\text { AST } \\
\mathrm{U} / 1\end{array}$ & $\begin{array}{l}\text { ALT } \\
\text { U/l }\end{array}$ \\
\hline Control (n = 3) & $69.7 \pm 3.6$ & $36.7 \pm 9.0$ & $1,066 \pm 34$ & $91.3 \pm 2.2$ & $63.8 \pm 7.1$ \\
\hline Nephritis $(\mathrm{n}=7)$ & $181.9 \pm 25.3^{*}$ & $104.0 \pm 15.7^{*}$ & $1,575 \pm 223$ & $87.4 \pm 7.8$ & $69.9 \pm 4.0$ \\
\hline Compound A $(n=7)$ & $95.9 \pm 18.3^{\#}$ & $62.9 \pm 15.1$ & $1,233 \pm 109$ & $91.4 \pm 3.2$ & $66.8 \pm 3.6$ \\
\hline
\end{tabular}

Plasma samples from unilaterally nephrectomized rats (Control), nephritic rats (Nephritis), and rats treated with compound A (Compound A) were collected. Data represent the mean and standard error. $\mathrm{TC}=$ Total cholesterol; $\mathrm{TG}=$ triglyceride. ${ }^{*} \mathrm{p}<0.05$ : significantly different from the control group. ${ }^{*} \mathrm{p}<$ 0.05 : significantly different from the nephritis group.

Tissue weights of the kidney, liver, spleen, and adrenal were increased in nephritic rats, and treatment with compound A ameliorated hypertrophy of the kidney, liver, and spleen (table 1). The elevated values of total cholesterol, triglyceride, and NEFA in nephritic rats were lower in the compound A-treated animals (table 2). There were little changes in AST and ALT levels in the nephritic and compound A-treated rats (table 2). These results suggest that compound A exhibited a renoprotective action without inducing hepatic injury.

\section{Effects of Compound A on Matrix-Related Gene Expressions}

To further analyze the effect of compound A on ECM deposition, changes of gene expression of $\alpha 1(\mathrm{I}), \alpha 1(\mathrm{III})$, and $\alpha 1$ (IV) collagen as well as of TGF- $\beta 1$ were examined (fig. $4 \mathrm{a}-$ d). The expressions of $\alpha 1(\mathrm{I}), \alpha 1(\mathrm{III}), \alpha 1(\mathrm{IV})$, and TGF- $\beta 1$ were apparently upregulated in the renal cortex of nephritic rats. Treatment with compound A significantly decreased the elevation of these gene expressions.

We have previously demonstrated that a change in the expression of MMP-2 was involved in the progress of chronic anti-Thyl.1 glomerulonephritis. Therefore, gene expression of MMP-2 in glomeruli was measured at the end of the study (fig. 4e). MMP-2 expression was increased in the nephritis group, as previously reported. Treatment with compound A suppressed the increase in MMP-2 gene expression. 


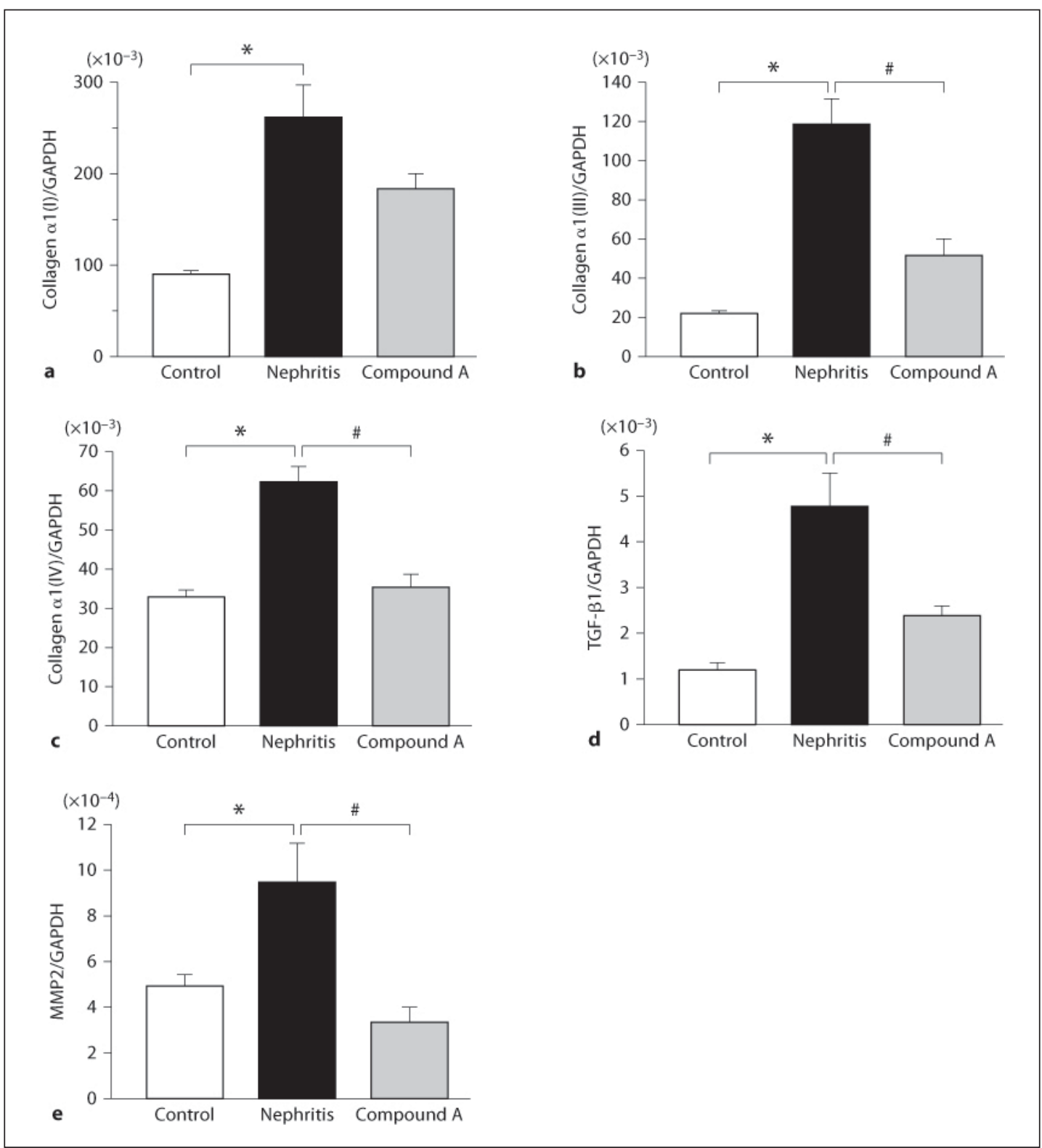

Fig. 4. Effect of compound A on gene expressions in nephritic rats. Quantitative analysis of gene expressions by real-time PCR for $\alpha 1$ (I) collagen (a), $\alpha 1$ (III) collagen (b), $\alpha 1$ (IV) collagen (c), TGF- $\beta 1$ (d), and MMP-2 (e). Columns show the mean values and bars the standard errors. White columns: unilaterally nephrectomized control rats $(n=3)$; black columns: nephritic rats $(n=4)$; gray columns: rats treated with compound A $(\mathrm{n}=4)$. ${ }^{*} \mathrm{p}<0.05$ : significantly different from the control group. ${ }^{\#} \mathrm{p}<0.05$ : significantly different from the nephritic group.

\section{Effects of Compound A on Injury of the GBM and GECs}

To further study GBM injury, the effect of compound A on the number of ACSs on the GBM was evaluated. Figure 5 shows the typical electron micrograph of glomerular capillary walls after polyethyleneimine perfusion. In control rats, electron-dense deposits consisting of the heparan sulfate-polyethyleneimine complex were distributed as linear arrays along the GBM (fig. 5a). Foot processes of GECs were regularly distributed along the GBM. In nephrit- 

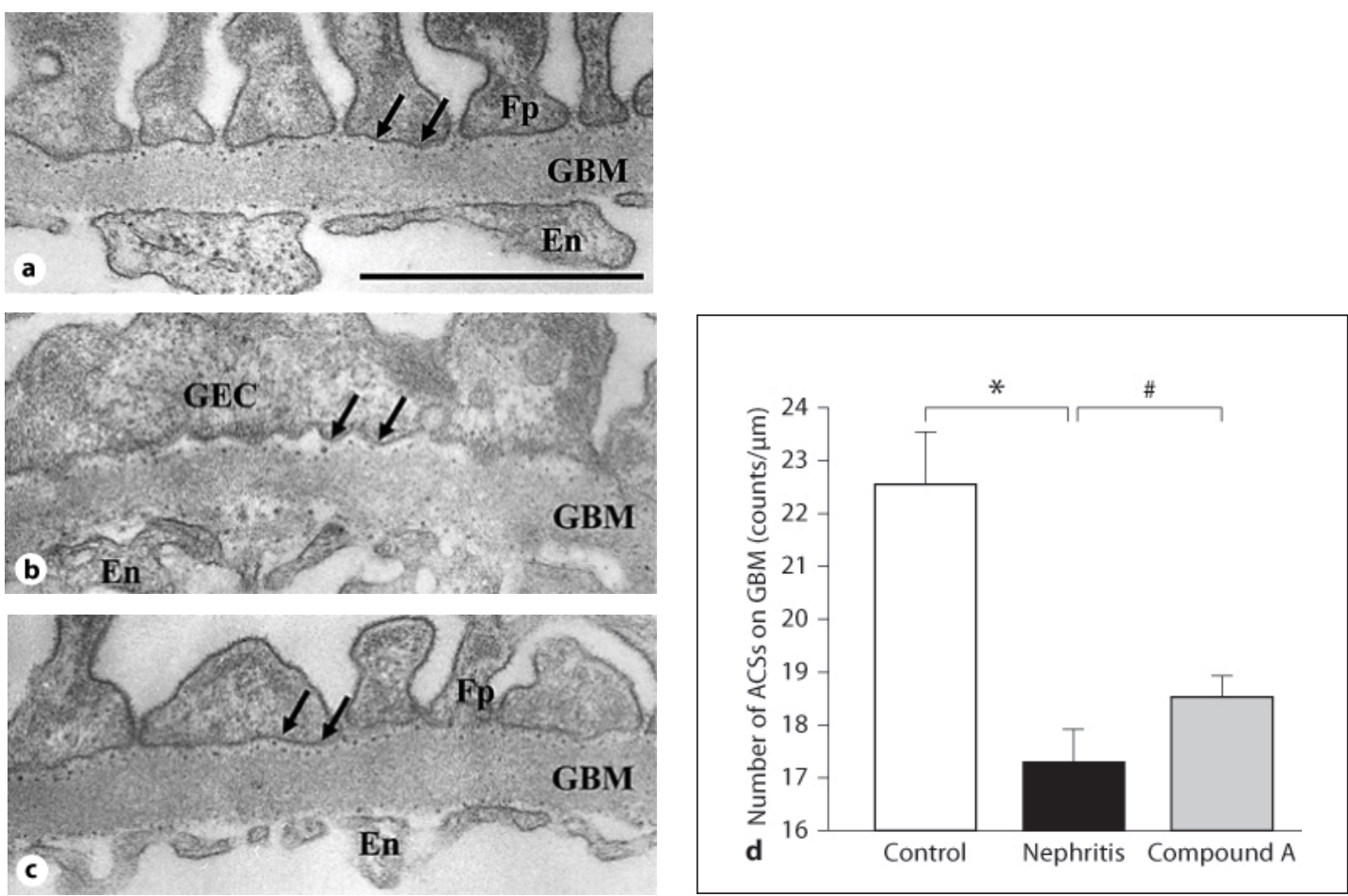

Fig. 5. ACS analysis. Electron micrographs of the parts of glomerular capillary walls perfused with polyethyleneimine at the end of the study. a Unilaterally nephrectomized control rat. ACSs on the GBM were distributed at a constant distance. $\mathbf{b}$ Nephritic rat. GECs are flat and their foot processes are effaced after the induction of nephritis. The number of ACSs on the GBM was reduced. $\mathbf{c}$ Rat treated with compound A. Drug treatment improved the alteration of cell form of GECs and prevented the decrease in the number of ACSs on the GBM. En = Endothelial cell; Fp = foot process. Arrows indicate ACSs. Bar $=1 \mu \mathrm{m}$. d The number of ACSs on the GBM. ACSs on the GBM stained with polyethyleneimine were evaluated by counting the number of dense deposits along the GBM and expressed as the number per 1,000 nm length of the GBM. Columns show the mean values and bars the standard errors. White column: unilaterally nephrectomized control rats $(n=3)$; black column: nephritic rats $(n=4)$; gray column: rats treated with compound A $(n=4) .{ }^{*} \mathrm{p}<0.05$ : significantly different from the control group. ${ }^{\#} \mathrm{p}<0.05$ : significantly different from the nephritis group.

ic rats, the number of stained ACSs was prominently decreased, and the effacement of foot processes was widely observed (fig. 5b). On the other hand, treatment with compound A maintained the number of ACSs and alleviated abnormal fusion of foot processes on the GMB (fig. 5c). Figure 5d shows the protective effect of compound A on the number of ACSs in each group at the end of the study.

To further analyze the action of compound A in GEC damage, immunostaining for desmin was performed as a marker for GEC injury. Staining for desmin was rarely observed in the control glomeruli (fig. 6a). In nephritic rats, an apparent expression of desmin was noted, and the staining score was significantly high (fig. 6b, d). In contrast, the expression of glomerular desmin was clearly less intense and decreased in rats treated with compound A (fig. 6c, d). 

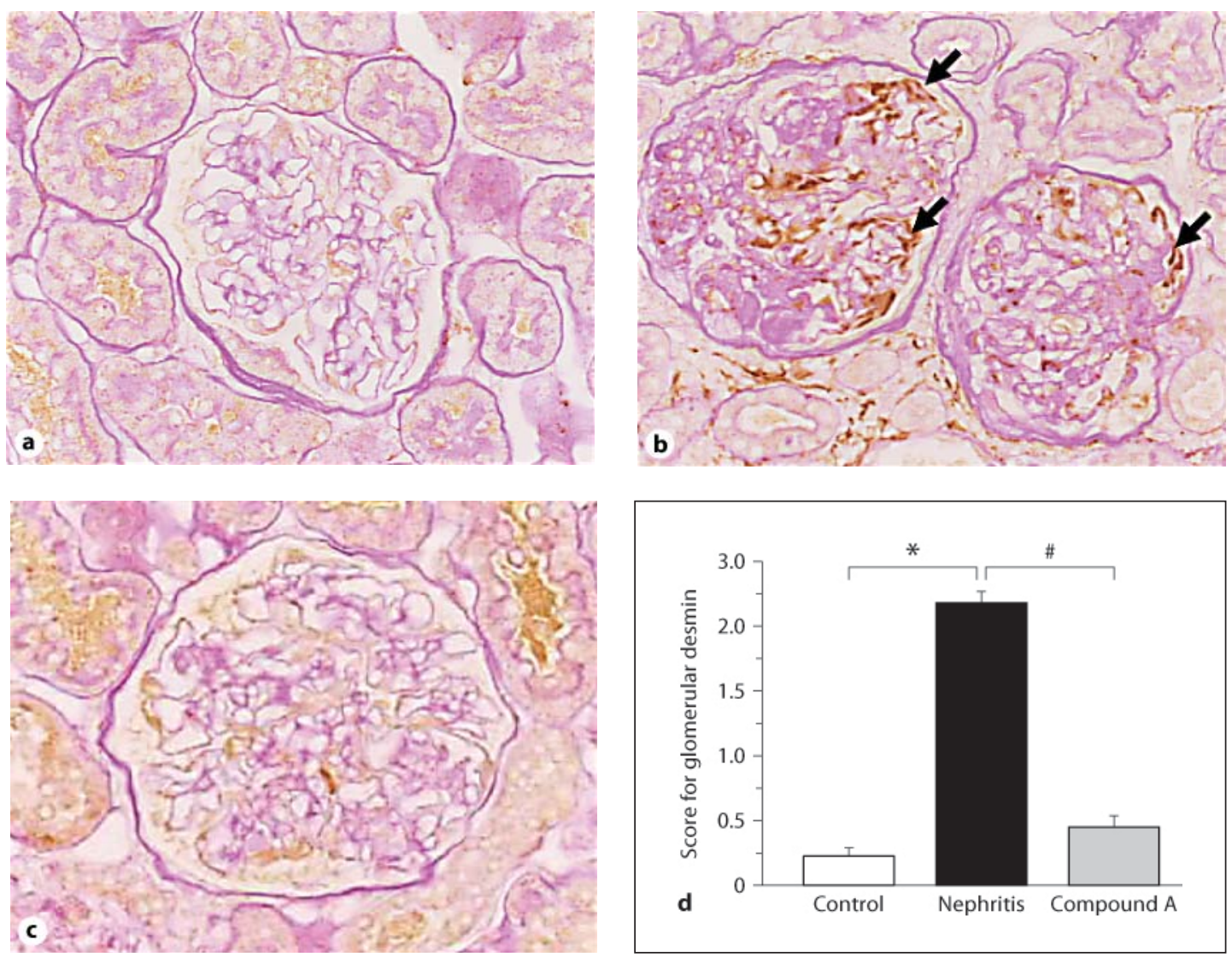

Fig. 6. Immunostaining for desmin. Unilaterally nephrectomized control rat (a), nephritic rat (b), and rat treated with compound A (c). Arrows indicate desmin deposition (b). d Semiquantitative analysis of the degree of staining for glomerular desmin. Columns show the mean values and bars the standard errors. White column: unilaterally nephrectomized control rats $(n=3)$; black column: nephritic rats $(n=4)$; gray column: rats treated with compound A $(n=4) .{ }^{*} \mathrm{p}<0.05$ : significantly different from the control group. ${ }^{\#} \mathrm{p}<0.05$ : significantly different from the nephritis group.

\section{Discussion}

In this study, we investigated the action of a novel MMP inhibitor, compound A, in chronic anti-Thyl.1 nephritis. There are previous reports that investigated the action of MMP inhibitors using anti-Thy1.1 nephritis. BB-1101, a non-selective MMP inhibitor, has been shown to induce a decrease in ECM deposition with improvement in massive proteinuria [24]. SI-27, a broad-spectrum inhibitor applied by subcutaneous implantation, significantly reduced glomerular cell number and suppressed collagen deposition [30]. However, these studies were based on acute nephritic models, with an interest in the accumulation of ECM and glomerular cell proliferation. In our previous study, we have shown that enhanced MMP-2 activity contributes to glomerular injury by altering the GBM component in chronic nephritic rats. This prompted us to study the effect of an MMP inhibitor on the damage of the GBM and GECs in an irreversible glomerulonephritic model.

GBM plays a central role in the control of the filtration function and the retention of the glomerular structure. The permeability of the GBM is defined as both size- and charge-selective properties. GECs cover the outer aspect of the GBM with extending foot processes 
and play a pivotal part in maintaining the stability of the glomerular tuft architecture and modulating GBM permeability. Therefore, damage of the GBM and GECs leads to an increase in proteinuria. We have previously reported sustained proteinuria in chronic antiThy1.1 nephritic rats, associated with a decreased number of ACSs on the GBM, an abnormal remodeling of type IV collagen in the GBM, and predominant injury in GECs $[16,27]$. The present study demonstrates that the treatment with the MMP inhibitor compound A prevented the loss of ACSs on the GBM. Furthermore, administration of compound A diminished abnormal fusion of foot processes and obviously prevented damage of GECs. These results show that the renoprotective effect of compound A on nephritis is associated with a relief of the deterioration in GBM constitution and function and conservation of GEC function. We have previously discussed that MMP-2 causes extensive structural remodeling during glomerular injury due to its possible involvement in the disruption of the GBM [16]. An abnormal structure of the GBM may rupture the contact with GECs, leading to GEC disorder. GEC damage, induced by puromycin aminonucleoside, results in the development of proteinuria with a decreased number of ACSs on the GBM [31]. Therefore, aberrant changes in GBM components and GECs evoked by the persistent activation of MMP-2 may be a pivotal part in the progression of chronic Thyl.1 nephritis.

Glomerulosclerosis and tubulointerstitial fibrosis are common features in end-stage kidney failure. In the process of kidney fibrosis, the balance between matrix synthesis and degradation shifts to favor accumulation of ECM [2]. Myofibroblasts identified by the expression of $\alpha$-smooth muscle actin contribute to the progression of both human and experimental nephropathy [32-37]. In sustained glomerular and tubulointerstitial scarring, MCs and TECs undergo phenotype changes and acquire myofibroblast properties, characterized by the increased production of interstitial matrix $[38,39]$. The presence of myofibroblasts in the development of glomerular and tubulointerstitial fibrosis has been demonstrated in antiThy1.1 nephritis $[23,40]$. It has been reported that MMP-2 contributes to the process of phenotype changes in MCs and TECs $[10,11,15]$. We have previously demonstrated that MMP2 activity is enhanced in anti-Thy1.1 nephritis [16]. Compound A obviously suppressed ECM deposition with decreased gene expressions of TGF- $\beta 1$ as well as $\alpha 1$ (I), $\alpha 1$ (III), and $\alpha 1$ (IV) collagen in the renal cortex. Therefore, our finding suggests that increased activity of MMP2 partly contributed to the progression of chronic anti-Thyl.1 nephritis, eventually leading to glomerulosclerosis and tubulointerstitial fibrosis. Furthermore, it is assumed that compound A might prevent phenotype changes in MCs and TECs to myofibroblasts by the inhibition of MMP-2 action. It has recently been discussed that the disruption of tubular basement membrane by MMP-2 induces the development of TEC phenotype changes, and selective MMP-2 inhibitors have a potential to treat CKD [12].

In conclusion, we have shown that the treatment with the synthetic MMP inhibitor compound A exhibited an ameliorative effect in an irreversible glomerulonephritic model. Our results indicate that activation of MMP-2 plays an important role in the progression of chronic nephritis underlying ECM accumulation in the glomeruli and tubulointerstitial area and impairment of GBM integrity and GECs. These findings suggest that inhibition of MMP-2 could be a potent approach to intervention in progressive glomerulonephritis. This hypothesis should be tested further, for instance using MMP-2-deficient mice. 


\section{References}

-1 Kamal A, Ahmed H: Matrix metalloproteinase and their inhibitors in kidney scarring: culprits or innocents. J Health Sci 2009;55:473-483.

-2 Schnaper HW: Balance between matrix synthesis and degradation: a determinant of glomerulosclerosis. Pediatr Nephrol 1995;9:104-111.

-3 Lenz O, Elliot SJ, Stetler-Stevenson WG: Matrix metalloproteinases in renal development and disease. J Am Soc Nephrol 2000;11:574-581.

-4 Bauvois B, Mothu N, Nguyen J, Nguyen-Khoa T, Nöel LH, Jungers P: Specific changes in plasma concentrations of matrix metalloproteinase-2 and -9, TIMP-1 and TGF-betal in patients with distinct types of primary glomerulonephritis. Nephrol Dial Transplant 2007;22:1115-1122.

-5 Thrailkill KM, Clay Bunn R, Fowlkes JL: Matrix metalloproteinases: their potential role in the pathogenesis of diabetic nephropathy. Endocrine 2009;35:1-10.

-6 Catania JM, Chen G, Parrish AR: Role of matrix metalloproteinases in renal pathophysiologies. Am J Physiol 2007;292:F905-F911.

-7 Ronco P, Chatziantoniou C: Matrix metalloproteinases and matrix receptors in progression and reversal of kidney disease: therapeutic perspectives. Kidney Int 2008;74:873-878.

-8 Butler GS, Butler MJ, Atkinson SJ, Will H, Tamura T, Schade van Westrum S, Crabbe T, Clements J, d'Ortho MP, Murphy G: The TIMP2 membrane type 1 metalloproteinase 'receptor' regulates the concentration and efficient activation of progelatinase A. A kinetic study. J Biol Chem 1998;273: $871-880$.

-9 Mazzieri R, Masiero L, Zanetta L, Monea S, Onisto M, Garbisa S, Mignatti P: Control of type IV collagenase activity by components of the urokinase-plasmin system: a regulatory mechanism with cellbound reactants. EMBO J 1997;16:2319-2332.

-10 Cheng S, Lovett DH: Gelatinase A (MMP-2) is necessary and sufficient for renal tubular cell epithelial-mesenchymal transformation. Am J Pathol 2003;162:1937-1949.

-11 Cheng S, Pollock AS, Mahimkar R, Olson JL, Lovett DH: Matrix metalloproteinase 2 and basement membrane integrity: a unifying mechanism for progressive renal injury. FASEB J 2006;20:18981900.

-12 Aresu L, Benali S, Garbisa S, Gallo E, Castagnaro M: Matrix metalloproteinases and their role in the renal epithelial mesenchymal transition. Histol Histopathol 2011;26:307-313.

-13 Lovett DH, Johnson RJ, Marti HP, Martin J, Davies M, Couser WG: Structural characterization of the mesangial cell type IV collagenase and enhanced expression in a model of immune complexmediated glomerulonephritis. Am J Pathol 1992;141:85-98.

-14 Jalalah SM, Furness PN, Barker G, Thomas M, Hall LL, Bicknell GR, Shaw JA, Pringle JH: Inactive matrix metalloproteinase 2 is a normal constituent of human glomerular basement membrane. An immuno-electron microscopic study. J Pathol 2000;191:61-66.

-15 Turck J, Pollock AS, Lee LK, Marti HP, Lovett DH: Matrix metalloproteinase 2 (gelatinase A) regulates glomerular mesangial cell proliferation and differentiation. J Biol Chem 1996;271:15074-15083.

-16 Kuroda T, Tanioka H, Masui M, Ito M, Notoya M, Shinosaki T: Altered expression of matrix-related molecules in the development of chronic Thy1.1 nephritis. Nephron Exp Nephrol 2006;104:e169e182.

-17 Lods N, Ferrari P, Frey FJ, Kappeler A, Berthier C, Vogt B, Marti HP: Angiotensin-converting enzyme inhibition but not angiotensin II receptor blockade regulates matrix metalloproteinase activity in patients with glomerulonephritis. J Am Soc Nephrol 2003;14:2861-2872.

-18 Chang HR, Yang SF, Li ML, Lin CC, Hsieh YS, Lian JD: Relationships between circulating matrix metalloproteinase-2 and -9 and renal function in patients with chronic kidney disease. Clin Chim Acta 2006;366:243-248.

19 Border WA, Noble NA: TGF- $\beta$ in kidney fibrosis: a target for gene therapy. Kidney Int 1997;51:13881396.

-20 Fukuda N, Tahira Y, Matsuda H, Matsumoto K: Transforming growth factor-beta as a treatment target in renal diseases. J Nephrol 2009;22:708-715.

-21 Sharma K, Ziyadeh FN: The emerging role of transforming growth factor-beta in kidney diseases. Am J Physiol 1994;266:F829-F842.

-22 Marti HP, Lee L, Kashgarian M, Lovett DH: Transforming growth factor-beta 1 stimulates glomerular mesangial cell synthesis of the 72-kd type IV collagenase. Am J Pathol 1994;144:82-94. 


\begin{tabular}{l|l}
\hline Nephron Extra 2012;2:133-146 \\
\hline DOI: $10.1159 / 000338801$ \\
Published online: May 26, 2012 & $\begin{array}{l}\text { @ 2012 S. Karger AG, Basel } \\
\text { www.karger.com/nne }\end{array}$ \\
\hline
\end{tabular}

Kuroda et al.: Renoprotective Action of a Matrix Metalloproteinase Inhibitor

-23 Lelongt B, Legallicier B, Piedagnel R, Ronco PM: Do matrix metalloproteinases MMP-2 and MMP-9 (gelatinases) play a role in renal development, physiology and glomerular diseases? Curr Opin Nephrol Hypertens 2001;10:7-12.

-24 Steinmann-Niggli K, Ziswiler R, Küng M, Marti HP: Inhibition of matrix metalloproteinases attenuates anti-Thyl.1 nephritis. J Am Soc Nephrol 1998;9:397-407.

-25 Shinosaki T, Notoya M, Nomura Y, Miyai I, Kobayashi T, Kurihara H: Glomerular epithelial cell injury accelerates the progression of antibody-induced mesangial proliferative nephritis. Exp Nephrol 2002;10:245-258.

-26 Maekawa R, Maki H, Wada T, Yoshida H, Nishida-Nishimoto K, Okamoto H, Matsumoto Y, Tsuzuki H, Yoshioka T: Anti-metastatic efficacy and safety of MMI-166, a selective matrix metalloproteinase inhibitor. Clin Exp Metastasis 2000;18:61-66.

-27 Shinosaki T, Miyai I, Nomura Y, Kobayashi T, Sunagawa N, Kurihara H: Mechanisms underlying the ameliorative property of lisinopril in progressive mesangioproliferative nephritis. Nephron 2002;91: 719-729.

-28 Kivirikko KI, Laitinen O, Prockop DJ: Modification of a specific assay for hydroxyproline in urine. Analytical Biochem 1967;19:249-255.

-29 Shinosaki T, Takagawa I, Sunagawa N, Yonetani Y, Kurihara H: Dual effect of lisinopril on puromycin aminonucleoside nephrosis in unilaterally nephrectomized rats. Jpn J Pharmacol 1997;73:337345.

-30 Mitani O, Katoh M, Shigematsu H: Participation of the matrix metalloproteinase inhibitor in Thy-1 nephritis. Pathol Int 2004;54:241-250.

- 31 Nakamura T, Ebihara I, Shirato I, Tomino Y, Koide H: Modulation of basement membrane component gene expression in glomeruli of aminonucleoside nephrosis. Lab Invest 1991;64:640-647.

-32 Alpers CE, Hudkins KL, Floege J, Johnson RJ: Human renal cortical interstitial cells with some features of smooth muscle cells participate in tubulointerstitial and crescentic glomerular injury. J Am Soc Nephrol 1994;5:201-209.

-33 Hewitson TD, Becker GJ: Interstitial myofibroblasts in IgA glomerulonephritis. Am J Nephrol 1995; 15:111-117.

-34 Essawy M, Soylemezoglu O, Muchaneta-Kubara EC, Shortland J, Brown CB, el Nahas AM: Myofibroblasts and the progression of diabetic nephropathy. Nephrol Dial Transplant 1997;12:43-50.

- 35 Ina K, Kitamura H, Tatsukawa S, Takayama T, Fujikura Y, Shimada T: Transformation of interstitial fibroblasts and tubulointerstitial fibrosis in diabetic nephropathy. Med Electron Microsc 2002;35: 87-95.

-36 Mandache E, Gherghiceanu M, Serafinceanu C, Penescu M, Mircescu G: Myofibroblast involvement in tubular basement membrane remodeling in type II diabetic nephropathy. Rom J Morphol Embryol 2011;52:75-79.

-37 Li J, Campanale NV, Liang RJ, Deane JA, Bertram JF, Ricardo SD: Inhibition of p38 mitogen-activated protein kinase and transforming growth factor-beta1/Smad signaling pathways modulates the development of fibrosis in adriamycin-induced nephropathy. Am J Pathol 2006;169:1527-1540.

- 38 Jefferson JA, Shankland SJ, Pichler RH: Proteinuria in diabetic kidney disease: a mechanistic viewpoint. Kidney Int 2008;74:22-36.

-39 Liu Y: New insights into epithelial-mesenchymal transition in kidney fibrosis. J Am Soc Nephrol 2010;21:212-222.

-40 Hugo C, Shankland SJ, Pichler RH, Couser WG, Johnson RJ: Thrombospondin 1 precedes and predicts the development of tubulointerstitial fibrosis in glomerular disease in the rat. Kidney Int 1998; 53:302-311. 